\title{
The specificity of color Doppler ultrasound to detect fetal hypoxia in pregnancy-induced-hypertension with thyroid dysfunction.
}

\author{
Xiao-dan Zhu, Shan-yu Yin, Bao-hua Wang, Tian-an Jiang* \\ Department of Ultrasonic Medicine, the First Hospital Affiliated of Zhejiang University School of Medicine, Hangzhou, \\ PR China
}

\begin{abstract}
The incidence of fetal hypoxia in pregnant women with Pregnancy-Induced Hypertension (PIH) is higher than normal pregnant women. Thyroid hormone is closely related to organism's metabolism. In this study, Color Doppler ultrasound was used to diagnose fetal hypoxia in pregnant women with PIH to further explore the correlation of hemodynamic indexes and thyroid hormone levels. 70 cases of pregnant women with PIH were divided into thyroid-normal group (39 cases), hyperthyroidism group (12 cases) and hypothyroidism group (19 cases). The hemodynamic indexes of Middle Cerebral Artery (MCA) and Umbilical Artery (UA) were detected by color Doppler ultrasound. In hyperthyroidism and hypothyroidism groups, the hemodynamic indexes of MCA were lower and hemodynamic indexes of UA were higher than thyroid-normal group. The results of correlation tests showed that, the hemodynamic indexes of UA and fetal hypoxia were both significantly correlated to thyroid hormone levels respectively, in pregnant women with PIH. Therefore, the hemodynamic index of UA may be an index which can predict thyroid function status in pregnant women with PIH. Pregnant women with PIH and thyroid dysfunction are more likely to get fetal hypoxia than those with normal thyroid function. Color Doppler ultrasound is sensitive to detect the changes.
\end{abstract}

Keywords: Color Doppler ultrasound, Fetal hypoxia, PIH, Thyroid.

Accepted on October 24, 2017

\section{Introduction}

Intrauterine fetus needs abundant oxygen to support normal growth and development. It is a complicated process in maternity, placenta and the fetus [1,2]. Therefore, any node going wrong in this process will cause fetal hypoxia. Fetal hypoxia can cause brain sparing effect that is characterized by the decreasing of hemodynamic index of MCA and increasing of cerebral blood flow in different extent [3-6]. Umbilical vessels are the only passageways linking fetus and placenta, the blood flow variation of which can reflect changes of placenta function [7]. The intrahepatic resistance in placenta increases when fetal hypoxia occurs. Therefore, Systolic/ Diastolic ratio (S/D), Pulsatility Index (PI) and Resistance Index (RI) in MCA and UA are used for determining fetal hypoxia by color Doppler ultrasound [8-10].

Pregnancy-Induced Hypertension (PIH) can reduce the bloodoxygen transfer area of placenta, destroy the balance of nutrient supply between maternity and placenta, and is far more likely to cause fetal hypoxia. Small arteries in spasticity is the primary reason to induce the elevation of blood pressure and further change cardiac and renal function, in pregnant women with PIH [11,12]. It seriously impacts on the blood supply to fetus. Production and metabolism of thyroid hormone in pregnant women with $\mathrm{PIH}$ are changed, because placenta produces large amounts of hormone and gestation changes cellular immune status. Therefore, hypothalamus-pituitarythyroid axis is put in a special stress state too [13]. Thyroid hormone is an indispensable hormone to maintain body's normal growth and development [14,15]. Gestation can result in thyroid gland dysfunction, in reverse, thyroid gland dysfunction can exacerbate PIH in pregnant women $[16,17]$. Fetal hypoxia and thyroid gland dysfunction were both studied deeply previously.

Now that thyroid gland dysfunction affects peripheral vascular resistance, whether the hemodynamic index of fetal hypoxia detected by color Doppler ultrasound could reflect the status of thyroid gland function on some levels? This paper expounded the problem in details.

\section{Materials and Methods}

\section{General information and different patient groups}

We selected 70 cases of pregnant women which had been diagnosed with PIH (BP $\geq 140 / 90 \mathrm{mmHg}$, urine protein of '-'), from 2013 to 2014, in the First Affiliated Hospital of Zhejiang Chinese Medical University. The average age of expectant mothers is $28.7 \pm 4.8 \mathrm{y}$ old and the average pregnant time is $32.3 \pm 4.7 \mathrm{w}$. The reference range of normal Thyroid 
Stimulating Hormone (TSH) levels within our hospital is 0.27-4.2 $\mathrm{mU} / \mathrm{L}$. According to thyroid hormone levels, 70 cases of pregnant women with PIH were divided into thyroid normal group (39 cases), hyperthyroidism group (12 cases) and hypothyroidism group (19 cases). All pregnant women involved were without diabetes, primary hypertension, congenital or acquired heart disease, chronic nephritis, ingestion of drugs or unspecified menstrual period. All fetuses did not have structural abnormalities of organs, genetic abnormalities, malformation, twins or multiple births. All voluntary pregnant women joined this research with informed consents. The study was approved by the review board of the independent ethics committee of the hospital.

\section{Color Doppler ultrasound}

All pregnant women were diagnosed by color Doppler ultrasound. The posture was adjusted to supine position and steady breath was maintained. The location of placenta was determined by color Doppler ultrasound with a probe of 2-4 MHZ and sampling volume of $2 \mathrm{~mm}$ (G60S, Siemens). During this process, the angle between sound beam and blood flow was $60^{\circ}$. After obstetrics routine practice, biparietal diameter of fetus was detected to obtain the transverse section of MCA, and intracranial color Doppler signals were caught and imaged by Color Doppler Flow Imaging (CDFI). Doppler sample volume was put in UA blood flow that the angles between sound beam and blood flow were $0-30^{\circ}$. When continuous 5 blood flow spectrums of MCA and UA were obtained by plus Doppler, S/D, RI and PI values of MCA and UA were determined. Each detecting time was less than $1 \mathrm{~min}$. All blood flow spectrums of pregnant women were performed 3 times, the average values was available to analysis.

\section{Statistical analyses}

Data were statistically analysed by SPSS 17.0 (SPSS, Inc., Chicago, IL, USA). Measurement data was presented at a format of mean \pm standard deviation. LSD t-test, chi-square test and one way analysis of variance were used in two or multiple groups to compare statistical differences. Correlation analyses were conducted in parametric comparison. $\mathrm{P}<0.05$ and $\mathrm{P}<0.01$ were considered to demonstrate statistically significant differences.

\section{Results}

\section{Comparation of hemodynamic indexes of $\mathrm{MCA}$ and $\mathrm{UA}$ in fetus of pregnant women with PIH}

The reference range of normal thyroid hormone levels in our hospital were 0.27-4.2. According to thyroid hormone levels, 70 cases of pregnant women with PIH involved were divided into thyroid-normal group, hyperthyroidism group and hypothyroidism group. The hemodynamic indexes of MCA and UA including RI, PI and S/D in fetus of pregnant women with PIH were determined by Color Doppler ultrasound. The results showed that RI, PI and S/D values of MCA in hyperthyroidism group were significantly lower than that of thyroid-normal group, and RI, PI and S/D values of UA in hyperthyroidism group were significantly higher than that of thyroid-normal group $(\mathrm{P}<0.05$, Table 1$)$. RI, PI and S/D of MCA in hypothyroidism group were significantly lower than that of thyroid-normal group, and RI, PI and S/D of UA in hypothyroidism group were significantly higher than that of thyroid-normal group $(\mathrm{P}<0.05$, Table 1$)$. Overall, no matter in hyperthyroidism group or hypothyroidism group, RI, PI and S/D values of MCA were decreased and RI, PI and S/D values of UA were increased, compared with thyroid-normal group.

\section{Determination of fetal hypoxia}

The clinical data showed that MCA RI $>0.6, \mathrm{PI}>1.6$ and $\mathrm{S} / \mathrm{D}>4$ and UA $\mathrm{S} / \mathrm{D}<3$ were found in fetus without intrauterine hypoxia, when detected by Color Doppler ultrasound. As shown in Table 2 and Figure 1, there were 9 cases of fetal hypoxia in 39 cases of PIH women with normal level of thyroid hormone, and 20 cases of fetal hypoxia in 31 cases of PIH women with abnormal level of thyroid hormone. The abnormality has been pointed by arrow. Significant differences existed among all groups $(\mathrm{P}<0.05$, Table 2$)$.
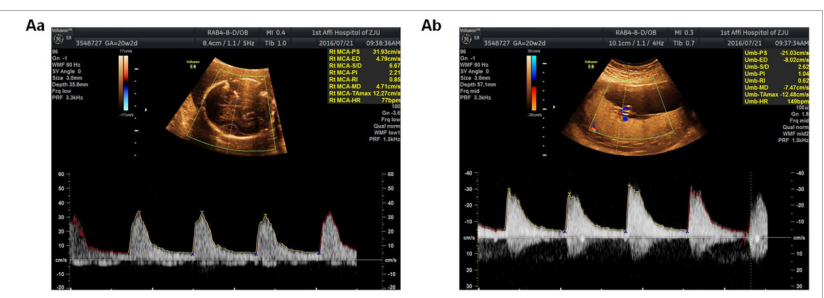

Ba
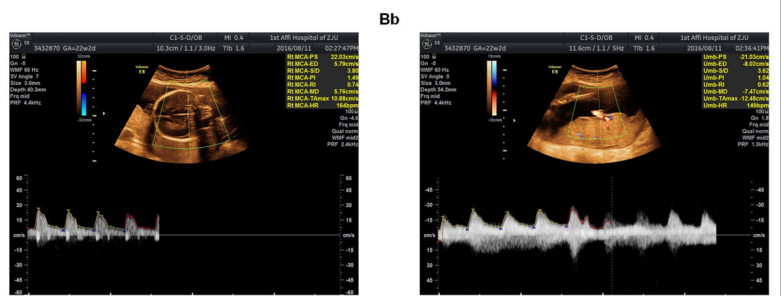

Figure 1. Color Doppler ultrasound images. (Aa) The fetus without intrauterine hypoxia of pregnant woman was detected by Color Doppler ultrasound in MCA. (Ab) The fetus without intrauterine hypoxia of pregnant woman was detected by Color Doppler ultrasound in UA. The abnormality has been pointed by arrow. (Ba) The fetus with intrauterine hypoxia of pregnant woman was detected by Color Doppler ultrasound in MCA. (Bb) The fetus with intrauterine hypoxia of pregnant woman was detected by Color Doppler ultrasound in UA. The abnormality has been pointed by arrow.

\section{Correlation analysis between thyroid hormone levels and fetal hypoxia}

As shown in Figure 2, the normal level of thyroid hormone had nothing to do with intrauterine hypoxia of fetus $(\mathrm{P}>0.05$; Figure 2A). Fetal hypoxia and hyperthyroidism presented significant negative correlation $(\mathrm{P}<0.05$; Figure $2 \mathrm{~B})$, while fetal hypoxia and hypothyroidism presented significant positive correlation $(\mathrm{P}<0.01$; Figure $2 \mathrm{C})$. In addition, the correlation of hypothyroidism and fetal hypoxia was higher 
The specificity of color Doppler ultrasound to detect fetal hypoxia in pregnancy-induced-hypertension with thyroid dysfunction

than the correlation of hyperthyroidism and fetal hypoxia (Figures 2B and 2C).
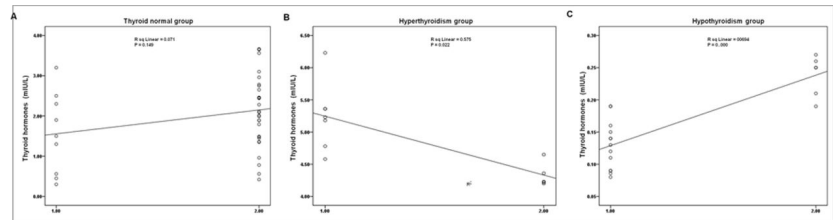

Figure 2. Correlation analyses between thyroid hormone levels and fetal hypoxia. (A) The normal level of thyroid hormone had nothing to do with intrauterine hypoxia of fetus. (B) The correlation analysis of fetal hypoxia and hyperthyroidism was evaluated. $(C)$ The correlation analysis of fetal hypoxia and hypothyroidism was evaluated. $P<0.05$ and $P<0.01$ were considered to demonstrate statistically significant differences.

\section{Correlation analysis between thyroid hormone levels of PIH women and S/D value of $U A$}

There were significant difference of thyroid hormone and S/D value of UA, between thyroid normal and hyperthyroidism groups, and between thyroid normal and hypothyroidism groups, respectively $(\mathrm{P}<0.05$; Table 3$)$. As shown in Figure 3, the normal level of thyroid hormone had nothing to do with $\mathrm{S} / \mathrm{D}$ value $(\mathrm{P}>0.05$; Figure $3 \mathrm{~A}) . \mathrm{S} / \mathrm{D}$ value and hyperthyroidism presented significant positive correlation $(\mathrm{P}<0.01$; Figure $3 \mathrm{~B})$, and as to hypothyroidism, it presented significant negative correlation $(\mathrm{P}<0.01$; Figure $3 \mathrm{C})$. In addition, the correlation of hypothyroidism and $\mathrm{S} / \mathrm{D}$ value was higher than the correlation of hyperthyroidism and S/D value (Figures $3 \mathrm{~B}$ and $3 \mathrm{C}$ ).

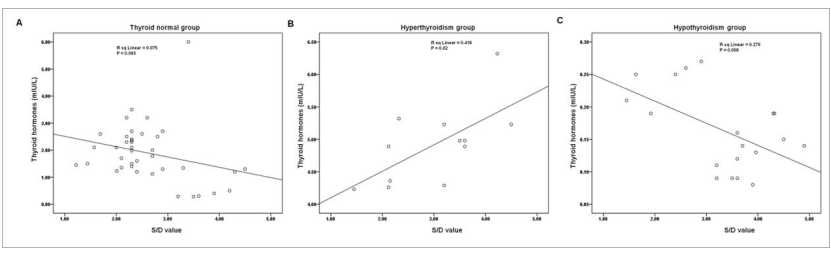

Figure 3. Correlation analyses between thyroid hormone levels of $P I H$ women and $S / D$ value of $U A$. (A) The correlation analysis of normal thyroid hormone level and $S / D$ value was evaluated. (B) The correlation analysis of hyperthyroidism and $S / D$ value was evaluated. (C) The correlation analysis of hypothyroidism and $S / D$ value was evaluated. $P<0.05$ and $P<0.01$ were considered to demonstrate statistically significant difference.

Table 1. Comparison of hemodynamic indexes of MCA and UA in thyroid normal, hyperthyroidism and hypothyroidism groups.

\begin{tabular}{|c|c|c|c|c|c|c|c|}
\hline \multirow[t]{2}{*}{ Group } & \multirow[t]{2}{*}{ Cases } & \multicolumn{3}{|l|}{ MCA } & \multicolumn{3}{|l|}{ UA } \\
\hline & & $\mathbf{R} \mathbf{I}$ & PI & S/D & $\mathbf{R} \mathbf{I}$ & PI & S/D \\
\hline Thyroid normal & 39 & $0.79 \pm 0.16$ & $1.86 \pm 0.15$ & $6.22 \pm 0.88$ & $0.65 \pm 0.23$ & $0.98 \pm 0.35$ & $3.12 \pm 0.19$ \\
\hline Hyperthyroidism & 12 & $0.65 \pm 0.07^{*}$ & $1.62 \pm 0.28^{*}$ & $4.38 \pm 0.12^{* *}$ & $0.77 \pm 0.31$ & $1.23 \pm 0.22$ & $3.44 \pm 0.25^{*}$ \\
\hline Hypothyroidism & 19 & $0.62 \pm 0.05^{*}$ & $1.49 \pm 0.11^{* *}$ & $4.02 \pm 0.19^{* *}$ & $0.83 \pm 0.09^{*}$ & $1.45 \pm 0.12^{* *}$ & $3.69 \pm 0.11^{* *}$ \\
\hline
\end{tabular}

${ }^{* *} \mathrm{P}<0.01$ and ${ }^{*} \mathrm{P}<0.05$ compared to thyroid normal group; one-way ANOVA

Table 2. Comparison of fetal hypoxia in thyroid normal, hyperthyroidism and hypothyroidism groups.

\begin{tabular}{llll}
\hline & $\begin{array}{l}\text { Gravida with fetal } \\
\text { hypoxia (cases) }\end{array}$ & $\begin{array}{l}\text { Gravida without fetal } \\
\text { hypoxia (cases) }\end{array}$ & P-value \\
\hline Thyroid normal & 9 & 30 & $\mathrm{P}=0.008$ \\
\hline Hyperthyroidism & 7 & 5 & \\
\hline Hypothyroidism & 13 & 6 & \\
\hline
\end{tabular}

Chi-square test, ${ }^{*} \mathrm{P}<0.05$

Table 3. Comparison of thyroid hormone levels of PIH women and $S / D$ values of $U A$ in thyroid normal, hyperthyroidism and hypothyroidism groups.

\begin{tabular}{lll}
\hline & $\begin{array}{l}\text { Thyroid } \\
\text { hormone } \\
\text { (mIU/L) }\end{array}$ & S/D value of UA \\
\hline Thyroid normal & $2.78 \pm 1.28$ & $3.12 \pm 0.19$ \\
\hline $\begin{array}{l}\text { Hyperthyroidis } \\
\mathrm{m}\end{array}$ & $4.36 \pm 2.01^{\star *}$ & $3.44 \pm 0.25^{*}$ \\
\hline Hypothyroidism & $0.15 \pm 0.07^{* *}$ & $3.69 \pm 0.11^{* *}$ \\
\hline
\end{tabular}

${ }^{* *} \mathrm{P}<0.01$ and ${ }^{*} \mathrm{P}<0.05$ compared to thyroid normal group; one-way ANOVA

\section{Discussion}

In PIH women, systemic arterioles suffering from convulsions and vascular permeability result in the increasing of plasma seepage flow and blood concentration $[12,13,18]$. PIH women are hypercoagulable to further induce fetal hypoxia caused by vascular occlusion $[12,19]$. Synthesized and secreted maternity thyroid hormones shall not only meet their own metabolic demands but also the metabolic demands of fetal central nervous system and placenta. Based on above evidences, $\mathrm{PIH}$ women are more prone to thyroid dysfunction than pregnant women without PIH [20]. In addition, thyroid dysfunction can exacerbate PIH and further increase the risk of fetal hypoxia. Therefore, color Doppler ultrasound was used to diagnose fetal hypoxia in pregnant women with PIH to further explore the correlation of hemodynamic indexes and thyroid hormone levels.

In general, placenta is gradually mature, chorionic vessels increase and get wider, placental resistance is reduced and blood flow is increased, during gestation process. The blood 
circulation in uteroplacental bed is in a state of low resistance and high flow rate $[21,22]$. S/D, PI and RI values in umbilical artery blood flow of fetus are taken as objective indexes to measure the peripheral resistance of placental to reflect fetalplacental circulation state in heamodynamics $[6,23,24]$. Cerebral blood vessels are in a state of compensatory enlargement and cerebral blood flow is accelerated to result in the decrease of hemodynamic indexes of MCA including S/D, PI and RI because of the increasing of vascular resistance caused by peripheral vessel contraction in fetal hypoxia. Therefore, in this study, the hemodynamic indexes of UA in conjunction with the hemodynamic indexes of MCA are considered to be more accurate to diagnose fetal hypoxia.

In our study, PIH women were divided into three groups according to thyroid hormone levels. Results showed that $\mathrm{S} / \mathrm{D}$, PI and RI values of MCA in PIH women with thyroid dysfunction were significantly lower than that of PIH women with normal thyroid function, S/D, PI and RI values of UA in PIH women with thyroid dysfunction had an opposite trend to that of MCA (Table 1). According to our clinical data and general standards $[6,8,23,24], \mathrm{PI}<1.6, \mathrm{~S} / \mathrm{D}<4$ and $\mathrm{RI}<0.6$ in MCA and $\mathrm{S} / \mathrm{D} \geq 3$ in UA were diagnosed as fetal hypoxia when detected by Color Doppler ultrasound. Our further analysis showed that there was significant difference between fetal hypoxia and thyroid hormone levels (Table 2 and Figure 1). In addition, fetal hypoxia correlated significantly with hyperthyroidism and hypothyroidism (Figure 2). It showed that the high or low levels of thyroid hormone in PIH women could affect circulatory resistance of bloodstream in fetus and were closely related to fetal hypoxia.

When vascular resistance grows, the blood flow decreases. The ratio of blood flow velocity in systolic and diastole of umbilical artery flow (S/D) and resistance of blood flow are the most common Doppler indexes [6,23]. In this study, we further found the correlation between thyroid hormone levels and S/D value of UA in fetus of PIH women. The results showed that S/D value of UA and hyperthyroidism presented significant positive correlation, and as to hypothyroidism, it presented significant negative correlation (Figure 3). PIH women with hyperthyroidism are in a state of high metabolic expenditure and high stress to accelerate fetal-placental circulation [25]. PIH women with hypothyroidism are in an opposite state [26]. Therefore, these results indicated that $S / D$ value of $U A$, detected by Color Doppler ultrasound, could reflect thyroid hormone levels in PIH women.

In conclusion, thyroid dysfunction of PIH women significantly affected fetal-placental circulation, color Doppler ultrasound is highly specific to diagnose fetal hypoxia in PIH women with thyroid dysfunction.

\section{References}

1. Arto-Medrano Fde A. Physiopathology of fetal hypoxia. Acta Obstet Ginecol Hisp Lusit 1968; 16: 223-234.

2. Eslamian L, Tooba K. Doppler findings in intrapartum fetal distress. Acta Med Iran 2011; 49: 547-550.
3. Mari G, Deter RL. Middle cerebral artery flow velocity waveforms in normal and small-for-gestational-age fetuses. Am J Obstet Gynecol 1992; 166: 1262-1270.

4. Nozaki AM, Francisco RP, Fonseca ES, Miyadahira S, Zugaib M. Fetal hemodynamic changes following maternal betamethasone administration in pregnancies with fetal growth restriction and absent end-diastolic flow in the umbilical artery. Acta Obstet Gynecol Scand 2009; 88: 350-354.

5. Soothill PW, Nicolaides KH, Rodeck $\mathrm{CH}$, Campbell S. Effect of gestational age on fetal and intervillous blood gas and acid-base values in human pregnancy. Fetal Ther 1986; 1: 168-175.

6. van den Wijngaard JA, Groenenberg IA, Wladimiroff JW, Hop WC. Cerebral Doppler ultrasound of the human fetus. Br J Obstet Gynaecol 1989; 96: 845-849.

7. Aksoy U. Prenatal color Doppler sonographic evaluation of nuchal encirclement by the umbilical cord. J Clin Ultrasound 2003; 31: 473-477.

8. Azpurua H, Dulay AT, Buhimschi IA, Bahtiyar MO, Funai E, Abdel-Razeq SS, Buhimschi CS. Fetal renal artery impedance as assessed by Doppler ultrasound in pregnancies complicated by intraamniotic inflammation and preterm birth. Am J Obstet Gynecol 2009; 200: 203. 201-211.

9. Liu F, Liu Y, Lai YP, Gu XN, Liu DM, Yang M. Fetal hemodynamics and fetal growth indices by ultrasound in late pregnancy and birth weight in gestational diabetes mellitus. Chin Med J (Engl) 2016; 129: 2109-2114.

10. Tarzamni MK, Nezami N, Sobhani N, Eshraghi N, Tarzamni M, Talebi Y. Nomograms of Iranian fetal middle cerebral artery Doppler waveforms and uniformity of their pattern with other populations nomograms. BMC Pregnancy Childbirth 2008; 8: 50.

11. Du X, Yuan Q, Yao Y, Li Z, Zhang H. Hypopituitarism and successful pregnancy. Int J Clin Exp Med 2014; 7: 4660-4665.

12. McKenna D, Tharmaratnam S, Mahsud S, Dornan J. Ultrasonic evidence of placental calcification at 36 weeks gestation: maternal and fetal outcomes. Acta Obstet Gynecol Scand 2005; 84: 7-10.

13. Yamaguchi K, Mishina J, Mitsuishi C, Nakabayashi M, Nishida H. Neonatal hypoglycemia in infants with intrauterine growth retardation due to pregnancy-induced hypertension. Acta Paediatr Jpn 1997; 39: 48-50.

14. Gürel A, Dogantekin A, Ozkan Y, Aydın S. Serum apelin levels in patients with thyroid dysfunction. Int J Clin Exp Med 2015; 8: 16394-16398.

15. Hall DR, Odendaal HJ, Kirsten GF, Smith J, Grove D. Expectant management of early onset, severe preeclampsia: perinatal outcome. BJOG 2000; 107: 1258-1264.

16. Glinoer D. What happens to the normal thyroid during pregnancy? Thyroid 1999; 9: 631-635.

17. Yen PM, Sinha R. Cellular action of thyroid hormone. Endotext. South Dartmouth (MA) MDText.com, Inc. 2000. 


\section{dysfunction}

18. Tranquilli AL, Giannubilo SR, Bezzeccheri V, Garbati E. The relative weight of Doppler of the uterine artery and 24 $\mathrm{h}$ ambulatory blood pressure monitoring in predicting hypertension in pregnancy and preeclampsia. Acta Biomed Ateneo Parmense 2000; 71: 351-355.

19. Song K, Li M. Pregnancy-induced hypertension caused by all-trans retinoic acid treatment in acute promyelocytic leukemia. Oncol Lett 2015; 10: 364-366.

20. Leung AS, Millar LK, Koonings PP, Montoro M, Mestman JH. Perinatal outcome in hypothyroid pregnancies. Obstet Gynecol 1993; 81: 349-353.

21. Liao QP, Buhimschi IA, Saade G, Chwalisz K, Garfield RE. Regulation of vascular adaptation during pregnancy and post-partum: effects of nitric oxide inhibition and steroid hormones. Hum Reprod 1996; 11: 2777-2784.

22. Rurak D, Bessette NW. Changes in fetal lamb arterial blood gas and acid-base status with advancing gestation. Am J Physiol Regul Integr Comp Physiol 2013; 304: 908-916.

23. Degani S, Wiingaard JA, Wladimiroff JW. Assessment by Doppler ultrasound of cerebral blood flow in the human fetus. Harefuah 1987; 112: 427-430.

24. Konje JC, Abrams KR, Taylor DJ. Normative values of Doppler velocimetry of five major fetal arteries as determined by color power angiography. Acta Obstet Gynecol Scand 2005; 84: 230-237.

25. Patil-Sisodia K, Mestman JH. Graves hyperthyroidism and pregnancy: a clinical update. Endocr Pract 2010; 16: 118-129.

26. Blazer S, Moreh-Waterman Y, Miller-Lotan R, Tamir A, Hochberg Z. Maternal hypothyroidism may affect fetal growth and neonatal thyroid function. Obstet Gynecol 2003; 102: 232-241.

\section{*Correspondence to}

Tian-an Jiang

Department of Ultrasonic Medicine

The First Hospital Affiliated of Zhejiang University School of Medicine

PR China

Email: chenmy@zju.edu.cn 